\title{
The interior Euler-Lagrange operator in field theory ${ }^{\dagger}$
}

\author{
Jana Volná and Zbyněk Urban
}

\begin{abstract}
The paper is devoted to the interior Euler-Lagrange operator in field theory, representing an important tool for constructing the variational sequence. We give a new invariant definition of this operator by means of a natural decomposition of spaces of differential forms, appearing in the sequence, which defines its basic properties. Our definition extends the well-known cases of the Euler-Lagrange class (Euler-Lagrange form) and the Helmholtz class (Helmholtz form). This linear operator has the property of a projector, and its kernel consists of contact forms. The result generalizes an analogous theorem valid for variational sequences over 1-dimensional manifolds and completes the known heuristic expressions by explicit characterizations and proofs.
\end{abstract}

Keywords Interior Euler-Lagrange operator, jet, Lagrangian, Euler-Lagrange expressions, Helmholtz conditions, variational sequence

MSC $201035 \mathrm{~A} 15,58 \mathrm{~A} 10,58 \mathrm{~A} 20,70 \mathrm{G} 75$

\section{Introduction}

In 1989, the variational sequence theory on finite order jet spaces was introduced by Krupka [6], primarily for the purpose of study of basic variational objects and their local and global properties. Later, the variational sequence was analysed in the particular case of fibred manifolds with 1-dimensional base (fibred mechanics, Krupka [7]). Related concepts of global variational theory (especially Lepage forms and variational bicomplexes) were also studied, see Krupka [9], Vitolo [14], and references therein. In particular, it was discovered that the Euler-Lagrange mapping, assigning to a Lagrangian ( $n$-form) its Euler-Lagrange form $((n+1)$-form, its coefficients are the Euler-Lagrange expressions) is a globally defined morphism in the variational sequence. Another important result of the variational sequence theory is the concept of Helmholtz class, a globalization of the well-known Helmholtz variationality conditions. The elements of the variational sequence are classes of differential forms on the underlying jet space, representing all known variational objects, such as total derivatives, Lagrangians, Euler-Lagrange equations, variationality conditions. Since the variational sequence is a quotient sequence, there arises a natural problem of representing classes by different geometric objects, e.g. differential forms. A mapping, assigning to a class its chart representative is known as the (local) interior Euler-Lagrange operator. This operator was considered by several authors in different ways: Anderson [1] introduced the interior Euler-Lagrange operator by means of (local) differential operators within the variational bicomplex theory; Krbek and Musilová [4] applied the integration by parts procedure;

$\dagger$ This paper was prepared in relation to the meeting Variations on a Theme (A meeting to celebrate the 70th birthday of Demeter Krupka), 23-24 August 2012, Levoča, Slovakia. 
for further approaches see also Bauderon [2], Dedecker and Tulczyjew [3]. Uniqueness of the interior Euler-Lagrange operator in the context of the variational bicomplex on infinite jet spaces was studied by Mikulski [10].

On the other hand, Krupka and Šeděnková-Volná [8], [12] introduced the interior EulerLagrange operator by means of decomposition theory of spaces of contact forms, naturally appearing in the sequence. This approach resulted in an invariant construction of the operator. Our objective is to generalize the results of [8], obtained for variational sequences over 1-dimensional manifolds, to the case of $n$-dimensional base manifolds (field theory). This paper completes the results of the preprint Volná [13]. We give a new proof of the main theorem, characterizing basic properties of the interior Euler-Lagrange operator. Namely, we show that this $\mathbb{R}$-linear operator (a) preserves the classes of differential forms in the sequence, (b) has the kernel coinciding with the space of contact forms, and (c) has the property of a projector.

Our basic references on the variational sequence theory on finite order jet prolongations of fibred manifolds are Krupka [5, 7,9] and Šeděnková-Volná [11,12].

Throughout this paper, the standard multiindex notation as well as the Einstein summation convention are freely applied. The symbol $i_{\xi} \rho$ denotes the contraction of a differential form $\rho$ by a vector field $\xi$.

\section{Background}

Throughout this paper we denote by $Y$ a fibred manifold over $n$-dimensional base $X$ with projection $\pi$, where $m=\operatorname{dim} Y-n$. Let $r \geq 0$. Let $J^{r} Y$ denote the $r$-jet prolongation of $Y$, and $\pi^{r, s}: J^{r} Y \rightarrow J^{s} Y, 0 \leq s<r$, and $\pi^{r}: J^{r} Y \rightarrow X$, denote the canonical jet projections. An element of $J^{r} Y$, denoted by $J_{x}^{r} \gamma$, is the $r$-jet of a section $\gamma$ of $\pi: Y \rightarrow X$ with source at a point $x \in X$. Recall that any fibred chart $(V, \psi), \psi=\left(x^{i}, y^{\sigma}\right)$, on $Y$, with $1 \leq i \leq n, 1 \leq \sigma \leq m$, induces the associated charts $\left(V^{r}, \psi^{r}\right), \psi^{r}=\left(x^{i}, y^{\sigma}, y_{j_{1}}^{\sigma}, y_{j_{1} j_{2}}^{\sigma}, \ldots, y_{j_{1} j_{2} \ldots j_{r}}^{\sigma}\right)$, on $J^{r} Y$, and $(U, \varphi), \varphi=\left(x^{i}\right)$, on $X$, where $V^{r}=\left(\pi^{r, 0}\right)^{-1}(V), U=\pi(V)$. The $r$-jet prolongation of a section $\gamma$ of $\pi$ is a section $J^{r} \gamma$ of $\pi^{r}$, defined by $J^{r} \gamma(x)=J_{x}^{r} \gamma$.

For an open set $W \subset Y$, we put $W^{r}=\left(\pi^{r, 0}\right)^{-1}(W)$. If $f: W^{r} \rightarrow \mathbb{R}$ is a function, then for any fibred chart $(V, \psi)$ such that $V \subset W$, we denote by $d_{i} f: V^{r+1} \rightarrow \mathbb{R}$ the $i$-th formal derivative of $f$ with respect to $(V, \psi)$; in fibred coordinates $\psi=\left(x^{i}, y^{\sigma}\right)$,

$$
d_{i} f=\frac{\partial f}{\partial x^{i}}+\sum_{l=0}^{r} \sum_{j_{1} \leq j_{2} \leq \ldots \leq j_{l}} \frac{\partial f}{\partial y_{j_{1} j_{2} \ldots j_{l}}^{\sigma}} y_{j_{1} j_{2} \ldots j_{l}}^{\sigma} \text {. }
$$

Recall that a vector field $\Xi$ on $Y$ is called $\pi$-vertical, if $T \pi \cdot \Xi=0$; this means in local coordinates $\Xi=\Xi^{\sigma}\left(\partial / \partial y^{\sigma}\right)$. The s-jet prolongation of a $\pi$-vertical vector field $\Xi$ on $Y$ is a vector field $J^{s} \Xi$ on $J^{s} Y$, given by

$$
J^{s} \Xi=\sum_{|J|=0}^{s} \Xi_{J}^{\sigma} \frac{\partial}{\partial y_{J}^{\sigma}}, \quad \text { where } \quad \Xi_{K i}^{\sigma}=d_{i} \Xi_{K}^{\sigma} .
$$

In fibred coordinates $\psi=\left(x^{i}, y^{\sigma}\right)$, we denote

$$
\begin{aligned}
& \omega_{0}=d x^{1} \wedge d x^{2} \wedge \ldots \wedge d x^{n}, \\
& \omega_{i}=i_{\partial / \partial x^{i}} \omega_{0}=(-1)^{i-1} d x^{1} \wedge d x^{2} \wedge \ldots \wedge d x^{i-1} \wedge d x^{i+1} \wedge \ldots \wedge d x^{n} .
\end{aligned}
$$

We say that a differential $k$-form $\rho$ on $J^{r} Y$ is contact, if it vanishes along the $r$-jet prolongation $J^{r} \gamma$ of every section $\gamma$ of $\pi$. An important case are contact 1 -forms; if $(V, \psi), \psi=\left(x^{i}, y^{\sigma}\right)$, is a fibred chart on $Y$, then a 1-form is contact if and only if it is a linear combination of the forms

(1) $\omega_{J}^{\sigma}=d y_{J}^{\sigma}-y_{J i}^{\sigma} d x^{i}$, 
where $J=\left(j_{1}, \ldots, j_{s}\right), 0 \leq s \leq r-1,0 \leq j_{1}, \ldots, j_{s} \leq n$. We get the contact basis of linear forms on $V^{r}$, constituted by $d x^{i}, \omega_{J}^{\sigma}, d y_{I}^{\sigma}$, where $0 \leq|J| \leq r-1$ and $|I|=r$. A general differential $k$-form $\rho$ on $V^{r}$ is contact if and only if $\rho$ is generated by 1 -forms $\omega_{J}^{\sigma}$, and by 2 -forms $d \omega_{I}^{\sigma}$, with $0 \leq|J| \leq r-1,|I|=r-1$ (Krupka [6]). After the canonical lifting to $J^{r+1} Y$, every differential $k$-form $\rho$ on $J^{r} Y$ has a unique decomposition, expressed by the sum of $l$-contact components $p_{l} \rho$ of $\rho$,

$$
\left(\pi^{r+1, r}\right)^{*} \rho=\sum_{l=0}^{k} p_{l} \rho=h \rho+\sum_{l=1}^{k} p_{l} \rho
$$

where $h \rho=p_{0} \rho$ is a horizontal component of $\rho$, and a $k$-form $p_{l} \rho$ on $J^{r+1} Y$ contains exactly $l$-factors $\omega_{J}^{\sigma}$ of the form (1); see e.g. [9]. Note that if $\rho$ is a contact $k$-form, then also the exterior derivative $d \rho$ is contact, and the exterior product of two contact forms is again a contact form. This implies, in particular, that contact forms constitute a differential ideal in exterior algebra of differential forms, called the contact ideal.

By means of the previous definition of a contact form, it is evident that every $k$-form with $k \geq n+1$ would be contact. For the forms of degree $\geq n+1$, we apply a new definition of contactness.

Let $k \geq n+1$. A differential $k$-form $\rho$ on $J^{r} Y$ is said to be contact, if for every point of $J^{r} Y$ there exist a fibred chart $(V, \psi)$ on $Y$, an integer $s, s \geq r$, and a contact $(k-1)$-form $\eta$ on $V^{s}$ such that

(2) $p_{k-n}\left(\left(\pi^{s, r}\right)^{*} \rho-d \eta\right)=0$.

Condition (2) is equivalent to saying that $\left(\pi^{s, r}\right)^{*} \rho$ can be expressed in the form

(3) $\left(\pi^{s, r}\right)^{*} \rho=\mu+d \eta$,

where $\mu$ is a $k$-form on $V^{s}$ such that $p_{k-n} \mu=0$, and $\eta$ is a contact $(k-1)$-form on $V^{s}$. A $k$-form $\rho$, with $k \geq n+1$, satisfying $p_{k-n} \rho=0$, is called strongly contact.

Let $k \geq 0$. Denote by $\Omega_{k}^{r}$ the direct image of the sheaf of smooth $k$-forms over $J^{r} Y$ by the jet projection $\pi^{r, 0}$. We set $\Omega_{0, c}^{r}=\{0\}$, and

$$
\begin{aligned}
& \Omega_{k, c}^{r}=\operatorname{ker} h, \quad \text { for } 1 \leq k \leq n, \\
& \Omega_{k, c}^{r}=\operatorname{ker} p_{k-n}, \quad \text { for } n+1 \leq k \leq N, N=\operatorname{dim} J^{r} Y,
\end{aligned}
$$

and

(5) $\Theta_{k}^{r}=\Omega_{k, c}^{s}+d \Omega_{k-1, c}^{s}$,

where $k \geq 1$, and $d \Omega_{k-1, c}^{s}$ is the image sheaf of $\Omega_{k-1, c}^{s}$ by $d$. Formula (5) means that a form $\rho$ belongs to $\Theta_{k}^{r}$ if its canonical lift $\left(\pi^{s, r}\right)^{*} \rho$ possesses a decomposition (3). Note that for $2 \leq k \leq n$, $\Theta_{k}^{r}=\Omega_{k, c}^{r}$. For every open set $W \subset Y, \Omega_{k}^{r} W$ (resp. $\Omega_{k, c}^{r} W$ ) is the Abelian group of $k$-forms (resp. contact $k$-forms $(1 \leq k \leq n)$, strongly contact $k$-forms $(n+1 \leq k \leq N))$ on $W^{r}, d \Omega_{k-1, c}^{r} W$ is the Abelian group of forms which can be locally expressed as differentials of contact, resp. strongly contact $(k-1)$-forms on $W^{r}$, and $\Theta_{k}^{r} W$ is a subgroup of $\Omega_{k}^{r} W$ of contact forms. We get an exact sequence of sheaves of Abelian groups

(6) $0 \rightarrow \Theta_{1}^{r} \rightarrow \Theta_{2}^{r} \rightarrow \Theta_{3}^{r} \rightarrow \cdots \rightarrow \Theta_{M}^{r} \rightarrow 0$,

in which all arrows denote the exterior differentiation $d$, and $M=m\left(\begin{array}{c}n+r-1 \\ n\end{array}\right)+2 n-1$ (see Krupka [6]). Sequence (6) is a subsequence of the de Rham sequence of differential forms

(7) $0 \rightarrow \mathbb{R} \rightarrow \Omega_{0}^{r} \rightarrow \Omega_{1}^{r} \rightarrow \Omega_{2}^{r} \rightarrow \cdots \rightarrow \Omega_{N-1}^{r} \rightarrow \Omega_{N}^{r} \rightarrow 0$, 
where $N=\operatorname{dim} J^{r} Y$. The quotient sequence

(8) $0 \rightarrow \mathbb{R} \rightarrow \Omega_{0}^{r} \rightarrow \Omega_{1}^{r} / \Theta_{1}^{r} \rightarrow \cdots \rightarrow \Omega_{M}^{r} / \Theta_{M}^{r} \rightarrow \Omega_{M+1}^{r} \rightarrow \cdots \rightarrow \Omega_{N}^{r} \rightarrow 0$

is also exact. We call (8) the $r$-th order variational sequence on $J^{r} Y$. The class of a differential form $\rho \in \Omega_{k}^{r} W$ in the variational sequence (8) is denoted by $[\rho]$.

The quotient mappings $E: \Omega_{k}^{r} / \Theta_{k}^{r} \rightarrow \Omega_{k+1}^{r} / \Theta_{k+1}^{r}$ are defined by

(9) $E([\rho])=[d \rho]$,

and satisfy the condition $E^{2}=0$. The quotient mapping $E: \Omega_{n}^{r} / \Theta_{n}^{r} \rightarrow \Omega_{n+1}^{r} / \Theta_{n+1}^{r}$ coincides with the well-known Euler-Lagrange mapping, and $E: \Omega_{n+1}^{r} / \Theta_{n+1}^{r} \rightarrow \Omega_{n+2}^{r} / \Theta_{n+2}^{r}$ is the HelmholtzSonin mapping.

A Lagrangian of order $r$ is a $\pi^{r}$-horizontal $n$-form $\lambda$. In a fibred chart, we write

(10) $\lambda=\mathscr{L} \omega_{0}$,

where $\mathscr{L}$ is a function on $J^{r} Y$, called the Lagrange function.

Let $\rho$ be an $n$-form on $J^{r} Y$. A form $\rho$ is called a Lepage $n$-form if $p_{1} d \rho$ is a $\pi^{r+1,0}$-horizontal $(n+1)$-form. A Lepage form $\rho$ is called a Lepage equivalent of a Lagrangian $\lambda$ if $h \rho=\lambda$. It is known that in higher order mechanics, Lepage equivalents are uniquely determined by Lagrangians. We denote by $\theta_{\lambda}$ a Lepage equivalent of a Lagrangian $\lambda$. If $r=1, \theta_{\lambda}$ is, e.g., the well known Poincaré-Cartan form, if $r>1$, we have the generalized Poincaré-Cartan form. If $\lambda$ has a local expression (10), then

(11) $p_{1} d \theta_{\lambda}=E_{\sigma}(\mathscr{L}) \omega^{\sigma} \wedge \omega_{0}$

where

(12) $E_{\sigma}(\mathscr{L})=\sum_{l=0}^{r}(-1)^{l} d_{j_{1}} d_{j_{2}} \ldots d_{j_{l}} \frac{\partial \mathscr{L}}{\partial y_{j_{1} \ldots j_{l}}^{\sigma}}$.

The form (11) is called the Euler-Lagrange form with components (12), the Euler-Lagrange expressions.

\section{The Interior Euler-Lagrange Operator}

First, we formulate the following lemma on the structure of 1-contact $(n+1)$-forms.

Lemma 1 Let $\rho$ be a 1-contact $(n+1)$-form on $J^{r+1} Y$, expressed by

(13) $\rho=\sum_{|J|=0}^{r} A_{\sigma}^{J} \omega_{J}^{\sigma} \wedge \omega_{0}$.

Then there exist a 1-contact $\omega^{\sigma}$-generated $(n+1)$-form $I_{1} \rho$ on $J^{2 r+1} Y$, a 1 -contact $n$-form $J_{1} \rho$ on $J^{2 r} Y$, and a 2-contact $(n+1)$-form $K_{1} \rho$ on $J^{2 r+1} Y$ such that

(14) $\left(\pi^{2 r+1, r+1}\right)^{*} \rho=I_{1} \rho-d J_{1} \rho+K_{1} \rho$,

where

$$
\begin{aligned}
& I_{1} \rho=B_{\sigma} \omega^{\sigma} \wedge \omega_{0}, \quad J_{1} \rho=\sum_{|J|=0}^{r-1} B_{\sigma}^{J i} \omega_{J}^{\sigma} \wedge \omega_{i}, \\
& K_{1} \rho=\sum_{|J|=0}^{r-1}\left(p d B_{\sigma}^{J i}\right) \wedge \omega_{J}^{\sigma} \wedge \omega_{i},
\end{aligned}
$$


and

$$
\begin{aligned}
& B_{\sigma}=\sum_{s=0}^{r}(-1)^{s} d_{i_{1}} d_{i_{2}} \ldots d_{i_{s}} A_{\sigma}^{i_{1} i_{2} \ldots i_{s}} \\
& B_{\sigma}^{J i}=\sum_{q=1}^{r-k}(-1)^{q+1} d_{i_{k+2}} d_{i_{k+3}} \ldots d_{i_{k+q}} A_{\sigma}^{J i i_{k+2} i_{k+3} \ldots i_{k+q}},
\end{aligned}
$$

with $|J|=k, k=0,1, \ldots, r-1$.

Proof. Suppose that a 1-contact $(n+1)$-form $\rho$ has an expression (13). By a direct calculation we obtain for every multiindex $J$,

(17) $P_{\sigma}^{J i} \omega_{J i}^{\sigma} \wedge \omega_{0}=-d_{i} P_{\sigma}^{J i} \omega_{J}^{\sigma} \wedge \omega_{0}+\left(p d P_{\sigma}^{J i}\right) \wedge \omega_{J}^{\sigma} \wedge \omega_{i}-d\left(P_{\sigma}^{J i} \omega_{J}^{\sigma} \wedge \omega_{i}\right)$.

Applying condition (17) to the form $\rho$, we separate all terms containing $\omega^{\sigma} \wedge \omega_{0}$. Then

$$
\begin{aligned}
& \left(\pi^{2 r+1, r+1}\right)^{*} \rho=\sum_{s=0}^{r} A_{\sigma}^{i_{1} i_{2} \ldots i_{s}} \omega_{i_{1} i_{2} \ldots i_{s}}^{\sigma} \wedge \omega_{0} \\
& =A_{\sigma} \omega^{\sigma} \wedge \omega_{0}+\sum_{s=1}^{r}\left(-d_{i_{s}} A_{\sigma}^{i_{1} \ldots i_{s-1} i_{s}} \omega_{i_{1} \ldots i_{s-1}}^{\sigma} \wedge \omega_{0}\right. \\
& \left.+\left(p d A_{\sigma}^{i_{1} \ldots i_{s-1} i_{s}}\right) \wedge \omega_{i_{1} \ldots i_{s-1}}^{\sigma} \wedge \omega_{i_{s}}-d\left(A_{\sigma}^{i_{1} \ldots i_{s-1} i_{s}} \omega_{i_{1} \ldots i_{s-1}}^{\sigma} \wedge \omega_{i_{s}}\right)\right) \\
& =A_{\sigma} \omega^{\sigma} \wedge \omega_{0}+\sum_{s=1}^{r}\left(d_{i_{s-1}} d_{i_{s}} A_{\sigma}^{i_{1} \ldots i_{s-2} i_{s-1} i_{s}} \omega_{i_{1} \ldots i_{s-2}}^{\sigma} \wedge \omega_{0}\right. \\
& -\left(p d\left(d_{i_{s}} A_{\sigma}^{i_{1} \ldots i_{s-2} i_{s-1} i_{s}}\right)\right) \wedge \omega_{i_{1} \ldots i_{s-2}}^{\sigma} \wedge \omega_{i_{s-1}} \\
& +d\left(d_{i_{s}} A_{\sigma}^{i_{1} \ldots i_{s-2} i_{s-1} i_{s}} \omega_{i_{1} \ldots i_{s-2}}^{\sigma} \wedge \omega_{i_{s-1}}\right) \\
& \left.+\left(p d A_{\sigma}^{i_{1} \ldots i_{s-1} i_{s}}\right) \wedge \omega_{i_{1} \ldots i_{s-1}}^{\sigma} \wedge \omega_{i_{s}}-d\left(A_{\sigma}^{i_{1} \ldots i_{s-1} i_{s}} \omega_{i_{1} \ldots i_{s-1}}^{\sigma} \wedge \omega_{i_{s}}\right)\right) \\
& =\ldots \\
& =\sum_{s=0}^{r}(-1)^{s} d_{i_{1}} \ldots d_{i_{s-1}} d_{i_{s}} A_{\sigma}^{i_{1} \ldots i_{s-1} i_{s}} \omega^{\sigma} \wedge \omega_{0} \\
& +\sum_{s=1}^{r} p d\left(\sum_{q=1}^{s}(-1)^{q+1} d_{i_{s-q+2}} \ldots d_{i_{s}} A_{\sigma}^{i_{1} \ldots i_{s-q} i_{s-q+1} \ldots i_{s}}\right) \wedge \omega_{i_{1} \ldots i_{s-q}}^{\sigma} \wedge \omega_{i_{s-q+1}} \\
& -d\left(\sum_{q=1}^{s}(-1)^{q+1} d_{i_{s-q+2}} \ldots d_{i_{s}} A_{\sigma}^{i_{1} \ldots i_{s-q} i_{s-q+1} \ldots i_{s}} \omega_{i_{1} \ldots i_{s-q}}^{\sigma} \wedge \omega_{i_{s-q+1}}\right),
\end{aligned}
$$

and resummating the last expression over $k$ with $k=s-q, k=0,1, \ldots, r-1$, we obtain

$$
\begin{aligned}
& \left(\pi^{2 r+1, r+1}\right)^{*} \rho \\
& =\sum_{s=0}^{r}(-1)^{s} d_{i_{1}} \ldots d_{i_{s-1}} d_{i_{s}} A_{\sigma}^{i_{1} \ldots i_{s-1} i_{s}} \omega^{\sigma} \wedge \omega_{0} \\
& -d\left(\sum_{k=0}^{r-1} \sum_{q=1}^{r-k}(-1)^{q+1} d_{i_{k+2}} \ldots d_{i_{k+q}} A_{\sigma}^{i_{1} \ldots i_{k} i_{k+1} i_{k+2} \ldots i_{k+q}} \omega_{i_{1} \ldots i_{k}}^{\sigma} \wedge \omega_{i_{k+1}}\right)
\end{aligned}
$$




$$
\begin{aligned}
& +\sum_{k=0}^{r-1} p d\left(\sum_{q=1}^{r-k}(-1)^{q+1} d_{i_{k+2}} \ldots d_{i_{k+q}} A_{\sigma}^{i_{1} \ldots i_{k} i_{k+1} i_{k+2} \ldots i_{k+q}}\right) \wedge \omega_{i_{1} \ldots i_{k}}^{\sigma} \wedge \omega_{i_{k+1}} \\
& =B_{\sigma} \omega^{\sigma} \wedge \omega_{0}-d\left(\sum_{|J|=0}^{r-1} B_{\sigma}^{J i} \omega_{J}^{\sigma} \wedge \omega_{i}\right)+\sum_{|J|=0}^{r-1}\left(p d B_{\sigma}^{J i}\right) \wedge \omega_{J}^{\sigma} \wedge \omega_{i} \\
& =I_{1} \rho-d\left(J_{1} \rho\right)+K_{1} \rho
\end{aligned}
$$

as required.

In the following lemma we show that the form $I_{1} \rho$ in decomposition (14) of Lemma 1 is uniquely determined.

Lemma 2 Suppose $\rho$ is a 1-contact $(n+1)$-form on $J^{r+1} Y$, expressed by (13), such that

(18) $\left(\pi^{2 r+1, r+1}\right)^{*} \rho=\rho_{0}-d \rho^{\prime}+\rho^{\prime \prime}$,

where $\rho_{0}$ is a 1 -contact $\omega^{\sigma}$-generated $(n+1)$-form on $J^{2 r+1} Y, \rho^{\prime}$ is a 1-contact $n$-form on $J^{2 r} Y$, and $\rho^{\prime \prime}$ is a 2-contact $(n+1)$-form on $J^{2 r+1} Y$. Then

(19) $\rho_{0}=I_{1} \rho$

and

(20) $-d \rho^{\prime}+\rho^{\prime \prime}=-d J_{1} \rho+K_{1} \rho$,

where the forms $I_{1} \rho, J_{1} \rho$ and $K_{1} \rho$ are given by (15).

Proof. By assumption (18) and by Lemma 1, (14), we have two decompositions of the form $\rho$, hence

(21) $0=\left(\rho_{0}-I_{1} \rho\right)-d\left(\rho^{\prime}-J_{1} \rho\right)+\left(\rho^{\prime \prime}-K_{1} \rho\right)$,

where $\rho_{0}-I_{1} \rho$ is a 1 -contact $\omega^{\sigma}$-generated $(n+1)$-form on $J^{2 r+1} Y, \rho^{\prime}-J_{1} \rho$ is a 1 -contact $n$-form on $J^{2 r} Y$, and $\rho^{\prime \prime}-K_{1} \rho$ is a 2 -contact $(n+1)$-form on $J^{2 r+1} Y$. Let us denote

(22) $\rho_{0}-I_{1} \rho=M_{\sigma} \omega^{\sigma} \wedge \omega_{0}, \quad \rho^{\prime}-J_{1} \rho=\sum_{|J|=0}^{2 r-1} N_{\sigma}^{J, i} \omega_{J}^{\sigma} \wedge \omega_{i}$.

Now we apply the formula

$$
d\left(\omega_{J}^{\sigma} \wedge \omega_{i j}\right)=\omega_{J i}^{\sigma} \wedge \omega_{j}-\omega_{J j}^{\sigma} \wedge \omega_{i}
$$

where $\omega_{i j}=i_{\partial / \partial x^{j}} i_{\partial / \partial x^{i}} \omega_{0}$. We get

$$
\begin{aligned}
& N_{\sigma}^{j_{1} \ldots j_{s}, i} \omega_{j_{1} \ldots j_{s}}^{\sigma} \wedge \omega_{i} \\
& \quad=\left(\frac{1}{s+1}\left(N_{\sigma}^{j_{1} \ldots j_{s}, i}+N_{\sigma}^{i j_{2} \ldots j_{s}, j_{1}}+N_{\sigma}^{j_{1} i j_{3} \ldots j_{s}, j_{2}}+\cdots+N_{\sigma}^{j_{1} \ldots j_{s-1} i, j_{s}}\right)\right. \\
& \quad+\frac{1}{s+1}\left(N_{\sigma}^{j_{1} \ldots j_{s}, i}-N_{\sigma}^{i j_{2} \ldots j_{s}, j_{1}}\right)+\frac{1}{s+1}\left(N_{\sigma}^{j_{1} \ldots j_{s}, i}-N_{\sigma}^{j_{1} i j_{3} \ldots j_{s}, j_{2}}\right) \\
& \left.\quad+\cdots+\frac{1}{s+1}\left(N_{\sigma}^{j_{1} \ldots j_{s}, i}-N_{\sigma}^{j_{1} \ldots j_{s-1} i, j_{s}}\right)\right) \omega_{j_{1} \ldots j_{s}}^{\sigma} \wedge \omega_{i}
\end{aligned}
$$




$$
\begin{aligned}
& =\frac{1}{s+1}\left(N_{\sigma}^{j_{1} \ldots j_{s}, i}+N_{\sigma}^{i j_{2} \ldots j_{s}, j_{1}}+N_{\sigma}^{j_{1} j_{3} \ldots j_{s}, j_{2}}+\cdots+N_{\sigma}^{j_{1} \ldots j_{s-1} i, j_{s}}\right) \omega_{j_{1} \ldots j_{s}}^{\sigma} \wedge \omega_{i} \\
& +\frac{1}{s+1} N_{\sigma}^{j_{1} \ldots j_{s}, i} d\left(\omega_{j_{2} \ldots j_{s}}^{\sigma} \wedge \omega_{j_{1} i}\right)+\frac{1}{s+1} N_{\sigma}^{j_{1} \ldots j_{s}, i} d\left(\omega_{j_{1} j_{3} \ldots j_{s}}^{\sigma} \wedge \omega_{j_{2} i}\right) \\
& +\cdots+\frac{1}{s+1} N_{\sigma}^{j_{1} \ldots j_{s}, i} d\left(\omega_{j_{1} \ldots j_{s-1}}^{\sigma} \wedge \omega_{j_{s} i}\right) \\
& =\tilde{N}_{\sigma}^{j_{1} \ldots j_{s} i} \omega_{j_{1} \ldots j_{s}}^{\sigma} \wedge \omega_{i} \\
& +\frac{1}{s+1} d\left(N_{\sigma}^{j_{1} \ldots j_{s}, i} \omega_{j_{2} \ldots j_{s}}^{\sigma} \wedge \omega_{j_{1} i}\right)+\frac{1}{s+1} d\left(N_{\sigma}^{j_{1} \ldots j_{s}, i} \omega_{j_{1} j_{3} \ldots j_{s}}^{\sigma} \wedge \omega_{j_{2} i}\right) \\
& +\cdots+\frac{1}{s+1} d\left(N_{\sigma}^{j_{1} \ldots j_{s}, i} \omega_{j_{1} \ldots j_{s-1}}^{\sigma} \wedge \omega_{j_{s} i}\right) \\
& -\frac{1}{s+1} d\left(N_{\sigma}^{j_{1} \ldots j_{s}, i}\right) \wedge \omega_{j_{2} \ldots j_{s}}^{\sigma} \wedge \omega_{j_{1} i}-\frac{1}{s+1} d\left(N_{\sigma}^{j_{1} \ldots j_{s}, i}\right) \wedge \omega_{j_{1} j_{3} \ldots j_{s}}^{\sigma} \wedge \omega_{j_{2} i} \\
& -\cdots-\frac{1}{s+1} d\left(N_{\sigma}^{j_{1} \ldots j_{s}, i}\right) \wedge \omega_{j_{1} \ldots j_{s-1}}^{\sigma} \wedge \omega_{j_{s} i},
\end{aligned}
$$

where

$$
\tilde{N}_{\sigma}^{j_{1} \ldots j_{s} i}=\frac{1}{s+1}\left(N_{\sigma}^{j_{1} \ldots j_{s}, i}+N_{\sigma}^{i j_{2} \ldots j_{s}, j_{1}}+N_{\sigma}^{j_{1} i j_{3} \ldots j_{s}, j_{2}}+\cdots+N_{\sigma}^{j_{1} \ldots j_{s-1} i, j_{s}}\right) .
$$

Computing 1-contact part of the right-hand side of equation (21), we obtain

$$
\begin{aligned}
0 & =\rho_{0}-I_{1} \rho-p_{1} d\left(\rho^{\prime}-J_{1} \rho\right) \\
& =M_{\sigma} \omega^{\sigma} \wedge \omega_{0}-p_{1} d\left(N_{\sigma}^{J, i} \omega_{J}^{\sigma} \wedge \omega_{i}\right) \\
& =M_{\sigma} \omega^{\sigma} \wedge \omega_{0}+d_{i} \tilde{N}_{\sigma}^{J i} \omega_{J}^{\sigma} \wedge \omega_{0}+\tilde{N}_{\sigma}^{J i} \omega_{J i}^{\sigma} \wedge \omega_{0} \\
& -\frac{1}{s+1} d_{j_{1}} N_{\sigma}^{j_{1} \ldots j_{s}, i} \wedge \omega_{j_{2} \ldots j_{s} i}^{\sigma} \wedge \omega_{0}+\frac{1}{s+1} d_{i} N_{\sigma}^{j_{1} \ldots j_{s}, i} \wedge \omega_{j_{2} \ldots j_{s} j_{1}}^{\sigma} \wedge \omega_{0} \\
& -\frac{1}{s+1} d_{j_{2}} N_{\sigma}^{j_{1} \ldots j_{s}, i} \wedge \omega_{j_{1} j_{3} \ldots j_{s} i}^{\sigma} \wedge \omega_{0}+\frac{1}{s+1} d_{i} N_{\sigma}^{j_{1} \ldots j_{s}, i} \wedge \omega_{j_{1} j_{3} \ldots j_{s} j_{2}}^{\sigma} \wedge \omega_{0} \\
& -\ldots \\
& -\frac{1}{s+1} d_{j_{s}} N_{\sigma}^{j_{1} \ldots j_{s}, i} \wedge \omega_{j_{1} \ldots j_{s-1} i}^{\sigma} \wedge \omega_{0}+\frac{1}{s+1} d_{i} N_{\sigma}^{j_{1} \ldots j_{s}, i} \wedge \omega_{j_{1} \ldots j_{s-1} j_{s}}^{\sigma} \wedge \omega_{0},
\end{aligned}
$$

hence the coefficients $M_{\sigma}, N_{\sigma}^{J, i}$, and $\tilde{N}_{\sigma}^{J i}$ must satisfy the conditions

$$
\begin{aligned}
& M_{\sigma}+d_{i} \tilde{N}_{\sigma}^{i}=0, \\
& d_{i} \tilde{N}_{\sigma}^{J j i}+\tilde{N}_{\sigma}^{J j}+\frac{1}{|J|+2} d_{i}\left(N_{\sigma}^{J i, j}-N_{\sigma}^{J j, i}\right)=0, \quad 0 \leq|J| \leq 2 r-2, \\
& \tilde{N}_{\sigma}^{j_{1} \ldots j_{2 r}}=0 .
\end{aligned}
$$

From the second equation we express

$$
\tilde{N}_{\sigma}^{i}=-d_{j} \tilde{N}_{\sigma}^{i j}-\frac{1}{2} d_{j}\left(N_{\sigma}^{j, i}-N_{\sigma}^{i, j}\right),
$$

and put these functions to the first equation of (23) to obtain

$$
M_{\sigma}-d_{i} d_{j} \tilde{N}_{\sigma}^{i j}=0 .
$$

Note that the antisymmetric part of $\tilde{N}_{\sigma}^{i}$ vanishes because of the symmetry of $d_{i} d_{j}$. We can repeat 
this procedure until we use the last condition $\tilde{N}_{\sigma}^{j_{1} \ldots j_{2 r}}=0$. Finally we get

$M_{\sigma}=0$

hence $\rho_{0}=I_{1} \rho$. Condition (20) now directly follows from (21). This completes the proof.

The preceding lemma gives us uniqueness of $I_{1} \rho$. We extend the operator $I_{1}$, acting on 1contact $(n+1)$-forms, to operator $I_{k}$ that will be defined on $k$-contact $(n+k)$-forms. To this purpose, we use inductive definition.

Let $k>1$. Suppose $\rho$ is a $k$-contact $(n+k)$-form on $J^{r+1} Y$, and $\Xi_{1}, \Xi_{2}, \ldots, \Xi_{k}$ are arbitrary $\pi$-vertical vector fields on $Y$. We define a $k$-contact $(n+k)$-form $I_{k} \rho$ on $J^{2 r+1} Y$ by

$$
\begin{aligned}
& i_{J} 2 r+1 \Xi_{k} \ldots i_{J 2 r+1} \Xi_{2} i_{J} 2 r+1 \Xi_{1} I_{k} \rho \\
& =\frac{1}{k}\left(i_{J 2 r+1 \Xi_{k}} i_{J 2 r+1} \Xi_{k-1} \ldots i_{J 2 r+1 \Xi_{3}} i_{J 2 r+1 \Xi_{2}} I_{k-1}\left(i_{J+1} \Xi_{1} \rho\right)\right. \\
& -i_{J 2 r+1} \Xi_{k} i_{J 2 r+1} \Xi_{k-1} \ldots i_{J 2 r+1} \Xi_{3} i_{J 2 r+1} \Xi_{1} I_{k-1}\left(i_{J r+1} \Xi_{2} \rho\right) \\
& -i_{J 2 r+1} \Xi_{k} i_{J 2 r+1} \Xi_{k-1} \ldots i_{J 2 r+1} \Xi_{1} i_{J 2 r+1} \Xi_{2} I_{k-1}\left(i_{J r+1} \Xi_{3} \rho\right) \\
& -\ldots \\
& -i_{J 2 r+1} \Xi_{k} i_{J 2 r+1} \Xi_{1} \ldots i_{J 2 r+1} \Xi_{3} i_{j 2 r+1} \Xi_{2} I_{k-1}\left(i_{J r+1} \Xi_{k-1} \rho\right) \\
& \left.-i_{J^{2 r+1} \Xi_{1}} i_{J^{2 r+1} \Xi_{k-1}} \ldots i_{J^{2 r+1} \Xi_{3}} i_{J^{2 r+1} \Xi_{2}} I_{k-1}\left(i_{J^{r+1} \Xi_{k}} \rho\right)\right)
\end{aligned}
$$

and a $k$-contact $(n+k-1)$-form $J_{k} \rho$ on $J^{2 r} Y$ by

$$
\begin{aligned}
& i_{J 2 r \Xi_{k}} \ldots i_{J 2 r \Xi_{2}} i_{j 2 r \Xi_{1}} J_{k} \rho \\
& \quad=-\frac{1}{k}\left(i_{J 2 r \Xi_{k}} i_{J 2 r \Xi_{k-1}} \ldots i_{J 2 r \Xi_{3}} i_{J 2 r \Xi_{2}} J_{k-1}\left(i_{J j+1} \Xi_{1} \rho\right)\right. \\
& \quad-i_{J 2 r \Xi_{k}} i_{J 2 r \Xi_{k-1}} \ldots i_{J 2 r \Xi_{3}} i_{J 2 r \Xi_{1}} J_{k-1}\left(i_{J r+1} \Xi_{2} \rho\right) \\
& \quad-i_{J 2 r \Xi_{k}} i_{J 2 r \Xi_{k-1}} \ldots i_{J 2 r} i_{1} i_{J 2 r \Xi_{2}} J_{k-1}\left(i_{J+1} \Xi_{3} \rho\right) \\
& \quad-\ldots \\
& \quad-i_{J 2 r \Xi_{k}} i_{J 2 r \Xi_{1}} \ldots i_{J 2 r \Xi_{3}} i_{J 2 r \Xi_{2}} J_{k-1}\left(i_{J r+1} \Xi_{k-1} \rho\right) \\
& \left.\quad-i_{J 2 r \Xi_{1}} i_{J 2 r \Xi_{k-1}} \ldots i_{J 2 r \Xi_{3}} i_{J 2 r \Xi_{2}} J_{k-1}\left(i_{J r+1} \Xi_{k} \rho\right)\right) .
\end{aligned}
$$

Finally, we define $K_{k} \rho$ by

(26) $K_{k} \rho=p_{k+1} d J_{k} \rho$.

Now let $\eta$ be an arbitrary $(n+k)$-form on $J^{r} Y, k \geq 1$. Using the previous definition of $I_{k}$ we set

(27) $\mathscr{I} \eta=I_{k} p_{k} \eta$

An $\mathbb{R}$-linear mapping

$$
\mathscr{I}: \Omega_{n+k}^{r} W \rightarrow \Omega_{n+k}^{2 r+1} W
$$

defined by (27), is called the interior Euler-Lagrange operator (cf. Anderson [1], Krupka and Šeděnková-Volná [8], Krbek and Musilová [4]). The form $\mathscr{I} \eta$ is called the canonical representative of $\eta$.

For our proof of the main theorem, we need the following lemma.

Lemma 3 Let $k \geq 1$. Let $W \subset Y$ be an open set in a fibred manifold $Y$. Suppose that an 
$(n+k)$-form $\rho \in \Omega_{n+k}^{r} W$ is expressed in a fibred chart on $W$ as

(29) $\rho=d\left(A_{\sigma_{1} \ldots \sigma_{k}}^{I_{1} \ldots I_{k} i} \omega_{I_{1}}^{\sigma_{1}} \wedge \cdots \wedge \omega_{I_{k}}^{\sigma_{k}} \wedge \omega_{i}\right)$.

Then $\mathscr{I} \rho=0$.

Proof. We proceed by induction. First, let $k=1$. By a direct computation, we have

$$
p_{1} \rho=-d_{i} A_{\sigma}^{I i} \omega_{I}^{\sigma} \wedge \omega_{0}-A_{\sigma}^{I i} \omega_{I i}^{\sigma} \wedge \omega_{0} .
$$

Using definition (27) of $\mathscr{I}$, and Lemma 1, (15), we obtain

$$
\begin{aligned}
\mathscr{I} \rho & =I_{1}\left(p_{1} \rho\right)=-\left(I_{1}\left(d_{i} A_{\sigma}^{I i} \omega_{I}^{\sigma} \wedge \omega_{0}\right)+I_{1}\left(A_{\sigma}^{I i} \omega_{I i}^{\sigma} \wedge \omega_{0}\right)\right) \\
& =-\sum_{s=0}^{r-1}(-1)^{s} d_{i_{1}} d_{i_{2}} \ldots d_{i_{s}} d_{i} A_{\sigma}^{i_{1} i_{2} \ldots i_{s} i} \omega^{\sigma} \wedge \omega_{0} \\
& -\sum_{s=1}^{r}(-1)^{s} d_{i_{1}} d_{i_{2}} \ldots d_{i_{s}} A_{\sigma}^{i_{1} i_{2} \ldots i_{s}} \omega^{\sigma} \wedge \omega_{0}=0 .
\end{aligned}
$$

Suppose now $\mathscr{I} \rho=0$ for every $(n+k)$-form (29), where $k \geq 1$. We show that the assertion is valid also for $(n+k+1)$-forms. Let $\rho \in \Omega_{n+k+1}^{r} W$ be of the form (29). Thus

$$
\begin{aligned}
\rho & =d\left(A_{\sigma_{1} \ldots \sigma_{k+1}}^{I_{1} \ldots I_{k+1} i} \omega_{I_{1}}^{\sigma_{1}} \wedge \cdots \wedge \omega_{I_{k+1}}^{\sigma_{k+1}} \wedge \omega_{i}\right) \\
& =d A_{\sigma_{1} \ldots \sigma_{k+1}}^{I_{1} \ldots I_{k+1} i} \omega_{I_{1}}^{\sigma_{1}} \wedge \cdots \wedge \omega_{I_{k+1}}^{\sigma_{k+1}} \wedge \omega_{i}+A_{\sigma_{1} \ldots \sigma_{k+1}}^{I_{1} \ldots I_{k+1} i} d\left(\omega_{I_{1}}^{\sigma_{1}} \wedge \cdots \wedge \omega_{I_{k+1}}^{\sigma_{k+1}}\right) \wedge \omega_{i},
\end{aligned}
$$

and for the $(k+1)$-contact component of $\rho$ we get

$$
\begin{aligned}
p_{k+1} \rho & =(-1)^{k+1} d_{i} A_{\sigma_{1} \ldots \sigma_{k+1}}^{I_{1} \ldots I_{k+1} i} \omega_{I_{1}}^{\sigma_{1}} \wedge \cdots \wedge \omega_{I_{k+1}}^{\sigma_{k+1}} \wedge \omega_{0} \\
& +(-1)^{k+1}(k+1) A_{\sigma_{1} \sigma_{2} \ldots \sigma_{k+1}}^{I_{1} I_{2} \ldots I_{I_{1} i} i} \omega_{I_{1}}^{\sigma_{1}} \wedge \omega_{I_{2}}^{\sigma_{2}} \wedge \cdots \wedge \omega_{I_{k+1}}^{\sigma_{k+1}} \wedge \omega_{0} .
\end{aligned}
$$

By definitions of $\mathscr{I}$ and $I_{k+1}$ (27), (24), it is now sufficient to show that for an arbitrary vertical vector field $\Xi$ on $Y, I_{k}\left(i_{J^{r+1}} \Xi p_{k+1} \rho\right)$ vanishes. Computing the contraction of (30), we have

$$
\begin{aligned}
& i_{J^{r+1}} \Xi p_{k+1} \rho=(-1)^{k+1}(k+1) d_{i} A_{\sigma_{1} \sigma_{2} \ldots \sigma_{k+1}}^{I_{1} I_{2} \ldots I_{k+1} i} \Xi_{I_{1}}^{\sigma_{1}} \omega_{I_{2}}^{\sigma_{2}} \wedge \cdots \wedge \omega_{I_{k+1}}^{\sigma_{k+1}} \wedge \omega_{0} \\
& +(-1)^{k+1}(k+1) A_{\sigma_{1} \sigma_{2} \ldots \sigma_{k+1}}^{I_{1} I_{2} \ldots I_{k+1} i} \Xi_{I_{1} i}^{\sigma_{1}} \omega_{I_{2}}^{\sigma_{2}} \wedge \cdots \wedge \omega_{I_{k+1}}^{\sigma_{k+1}} \wedge \omega_{0} \\
& -(-1)^{k+1}(k+1) A_{\sigma_{1} \sigma_{2} \ldots \sigma_{k+1}}^{I_{1} I_{2} \ldots I_{k+1} i} \omega_{I_{1} i}^{\sigma_{1}} \wedge\left(k \Xi_{I_{2}}^{\sigma_{2}} \omega_{I_{3}}^{\sigma_{3}} \wedge \cdots \wedge \omega_{I_{k+1}}^{\sigma_{k+1}}\right) \wedge \omega_{0} \\
& =(-1)^{k+1}(k+1) d_{i}\left(A_{\sigma_{1} \sigma_{2} \ldots \sigma_{k+1}}^{I_{1} I_{2} \ldots I_{k+1} i} \Xi_{I_{1}}^{\sigma_{1}}\right) \omega_{I_{2}}^{\sigma_{2}} \wedge \cdots \wedge \omega_{I_{k+1}}^{\sigma_{k+1}} \wedge \omega_{0} \\
& +(-1)^{k+1}(k+1) A_{\sigma_{1} \sigma_{2} \sigma_{3} \ldots \sigma_{k+1}}^{I_{1} I_{2} I_{3} \ldots I_{k+1} i} k \Xi_{I_{1}}^{\sigma_{1}} \omega_{I_{2} i}^{\sigma_{2}} \wedge \omega_{I_{3}}^{\sigma_{3}} \wedge \cdots \wedge \omega_{I_{k+1}}^{\sigma_{k+1}} \wedge \omega_{0} \\
& =p_{k} \eta \text {, }
\end{aligned}
$$

where

$$
\eta=d\left(\tilde{A}_{\sigma_{2} \sigma_{3} \ldots \sigma_{k+1}}^{I_{2} I_{3} \ldots I_{k+1} i} \omega_{I_{2}}^{\sigma_{2}} \wedge \omega_{I_{3}}^{\sigma_{3}} \wedge \cdots \wedge \omega_{I_{k+1}}^{\sigma_{k+1}} \wedge \omega_{i}\right),
$$

and

$$
\tilde{A}_{\sigma_{2} \sigma_{3} \ldots \sigma_{k+1}}^{I_{2} I_{3} \ldots I_{k+1} i}=-(k+1) A_{\sigma_{1} \sigma_{2} \sigma_{3} \ldots \sigma_{k+1}}^{I_{1} I_{2} I_{3} \ldots I_{k+1} i} \Xi_{I_{1}}^{\sigma_{1}} .
$$

But by our assumption, $\mathscr{I} \eta=0$ hence $I_{k}\left(i_{J^{r+1} \Xi} p_{k+1} \rho\right)=I_{k}\left(p_{k} \eta\right)=0$, as required. 
The following theorem characterizes the main properties of the interior Euler-Lagrange operator $\mathscr{I}$.

Theorem 1 Let $k \geq 1$ be an integer. Let $\pi: Y \rightarrow X$ be a fibred manifold over $n$-dimensional base $X$, and $W \subset Y$ be an open set.

(a) For every $\rho \in \Omega_{n+k}^{r} W, \mathscr{I} \rho$ lies in the same class as $\left(\pi^{2 r+1, r}\right)^{*} \rho$.

(b) The kernel of $\mathscr{I}: \Omega_{n+k}^{r} W \rightarrow \Omega_{n+k}^{2 r+1} W$ coincides with $\Theta_{n+k}^{r} W$.

(c) $\mathscr{I} \circ \mathscr{I}=\mathscr{I}$ (up to the canonical jet projection).

Proof. (a) When it is obvious from the context, we omit pull-back of forms by the canonical jet projection $\pi^{2 r+1, r+1}$. We proceed by induction. If $k=1$, then $\mathscr{I} \rho-\rho \in \Theta_{n+1}^{2 r+1}$ is straightforward from decomposition (14) and formulas (15) of Lemma 1.

Suppose that $\mathscr{I} \rho-\rho \in \Theta_{n+k}^{2 r+1}$ for some $k>1$. We shall prove that the same condition holds for $k+1$. Let $\rho$ be an arbitrary $(n+k+1)$-form on $W^{r} \subset J^{r} Y$. From definition (27) we have $\mathscr{I} \rho=I_{k+1}\left(p_{k+1} \rho\right)$. For an arbitrary $\pi$-vertical vector field $\Xi$ on $Y, i_{J^{r+1}} \Xi p_{k+1} \rho$ is a $k$-contact $(n+k)$-form hence, by our assumption, $\mathscr{I}\left(i_{J^{r+1} \Xi} p_{k+1} \rho\right)-i_{J^{r+1} \Xi} p_{k+1} \rho$ belongs to $\Theta_{n+k}^{2 r+1} W$; we write

(31) $I_{k}\left(i_{J^{r+1}} p_{k+1} \rho\right)=i_{J^{r+1} \Xi} p_{k+1} \rho+\mu_{\Xi}$,

where $\mu_{\Xi} \in \Theta_{n+k}^{2 r+1} W$. Substituting (31) in the definition of $I_{k+1}$ (24), we get for arbitrary $\pi$ vertical vector fields $\Xi_{1}, \Xi_{2}, \ldots, \Xi_{k+1}$,

$$
\begin{aligned}
& i_{J 2 r+1} \Xi_{k+1} \ldots i_{J 2 r+1} \Xi_{2} i_{J 2 r+1} \Xi_{1} I_{k+1}\left(p_{k+1} \rho\right) \\
& =\frac{1}{k+1}\left(i_{J 2 r+1} \Xi_{k+1} i_{J 2 r+1} \Xi_{k} \ldots i_{J 2 r+1} \Xi_{3} i_{J 2 r+1} \Xi_{2} I_{k}\left(i_{J r+1} \Xi_{1} p_{k+1} \rho\right)\right. \\
& -i_{J^{2 r+1}} \Xi_{k+1} i_{J^{2 r+1} \Xi_{k}} \ldots i_{J 2 r+1} \Xi_{3} i_{J 2 r+1} \Xi_{1} I_{k}\left(i_{J r+1} \Xi_{2} p_{k+1} \rho\right) \\
& -i_{J} 2 r+1 \Xi_{k+1} i_{J} 2 r+1 \Xi_{k} \ldots i_{J 2 r+1} \Xi_{1} i_{J} 2 r+1 \Xi_{2} I_{k}\left(i_{J r+1} \Xi_{3} p_{k+1} \rho\right) \\
& -\ldots \\
& -i_{J 2 r+1 \Xi_{k+1}} i_{J 2 r+1} \Xi_{1} \ldots i_{J 2 r+1} \Xi_{3} i_{J 2 r+1} \Xi_{2} I_{k}\left(i_{J r+1} \Xi_{k} p_{k+1} \rho\right) \\
& \left.-i_{J 2 r+1} \Xi_{1} i_{J 2 r+1} \Xi_{k} \ldots i_{J 2 r+1} \Xi_{3} i_{J 2 r+1} \Xi_{2} I_{k}\left(i_{J r+1} \Xi_{k+1} p_{k+1} \rho\right)\right) \\
& =\frac{1}{k+1}\left(i_{J^{2 r+1} \Xi_{k+1}} i_{J^{2 r+1} \Xi_{k}} \ldots i_{J^{2 r+1} \Xi_{3}} i_{J^{2 r+1} \Xi_{2}}\left(i_{J r+1} \Xi_{1} p_{k+1} \rho+\mu_{\Xi_{1}}\right)\right. \\
& -i_{J^{2 r+1}} \Xi_{k+1} i_{J^{2 r+1} \Xi_{k}} \ldots i_{J 2 r+1} \Xi_{3} i_{J 2 r+1} \Xi_{1}\left(i_{J+1} \Xi_{2} p_{k+1} \rho+\mu_{\Xi_{2}}\right) \\
& -i_{J 2 r+1} \Xi_{k+1} i_{J 2 r+1} \Xi_{k} \ldots i_{J 2 r+1} \Xi_{1} i_{J 2 r+1} \Xi_{2}\left(i_{J r+1} \Xi_{3} p_{k+1} \rho+\mu_{\Xi_{3}}\right) \\
& -\ldots \\
& -i_{J 2 r+1 \Xi_{k+1}} i_{J 2 r+1} \Xi_{1} \ldots i_{J 2 r+1} \Xi_{3} i_{J 2 r+1 \Xi_{2}}\left(i_{J+1} \Xi_{k} p_{k+1} \rho+\mu_{\Xi_{k}}\right) \\
& \left.-i_{J 2 r+1} \Xi_{1} i_{J 2 r+1} \Xi_{k} \ldots i_{J 2 r+1} \Xi_{3} i_{J 2 r+1} \Xi_{2}\left(i_{J r+1} \Xi_{k+1} p_{k+1} \rho+\mu_{\Xi_{k+1}}\right)\right) \\
& =i_{J 2 r+1} \Xi_{k+1} \ldots i_{J 2 r+1} \Xi_{2} i_{J 2 r+1} \Xi_{1} p_{k+1} \rho+\frac{1}{k+1}\left(i_{J^{2 r+1} \Xi_{k+1}} i_{J^{2 r+1} \Xi_{k}} \ldots i_{J 2 r+1} \Xi_{3} i_{J 2 r+1} \Xi_{2}\left(\mu_{\Xi_{1}}\right)\right. \\
& -i_{J 2 r+1} \Xi_{k+1} i_{J 2 r+1} \Xi_{k} \ldots i_{J 2 r+1} \Xi_{3} i_{J 2 r+1} \Xi_{1}\left(\mu_{\Xi_{2}}\right) \\
& -i_{J 2 r+1} \Xi_{k+1} i_{J 2 r+1} \Xi_{k} \ldots i_{J 2 r+1} \Xi_{1} i_{J 2 r+1} \Xi_{2}\left(\mu_{\Xi_{3}}\right) \\
& -\ldots \\
& -i_{J 2 r+1 \Xi_{k+1}} i_{J 2 r+1} \Xi_{1} \ldots i_{J 2 r+1} \Xi_{3} i_{22 r+1 \Xi_{2}}\left(\mu_{\Xi_{k}}\right) \\
& \left.-i_{J 2 r+1} \Xi_{1} i_{J 2 r+1} \Xi_{k} \ldots i_{J 2 r+1} \Xi_{3} i_{J 2 r+1} \Xi_{2}\left(\mu_{\Xi_{k+1}}\right)\right) \text {, }
\end{aligned}
$$


and consequently

$$
\begin{aligned}
& i_{J^{2 r+1}} \Xi_{k+1} \ldots i_{J^{2 r+1} \Xi_{2}} i_{J^{2 r+1} \Xi_{1}}\left(I_{k+1}\left(p_{k+1} \rho\right)-p_{k+1} \rho\right) \\
& \quad=\frac{1}{k+1}\left(i_{J^{2 r+1} \Xi_{k+1}} i_{J^{2 r+1} \Xi_{k}} \ldots i_{J^{2 r+1} \Xi_{3}} i_{J^{2 r+1} \Xi_{2}}\left(\mu_{\Xi_{1}}\right)\right. \\
& \quad-i_{J^{2 r+1} \Xi_{k+1}} i_{J^{2 r+1} \Xi_{k}} \ldots i_{J^{2 r+1} \Xi_{3}} i_{J^{2 r+1} \Xi_{1}}\left(\mu_{\Xi_{2}}\right) \\
& \quad-\ldots \\
& \left.\quad-i_{J 2 r+1} \Xi_{1} i_{J^{2 r+1} \Xi_{k}} \ldots i_{J^{2 r+1} \Xi_{3}} i_{J^{2 r+1} \Xi_{2}}\left(\mu_{\Xi_{k+1}}\right)\right) .
\end{aligned}
$$

Let $\xi_{1}, \ldots, \xi_{n} \in T_{x} J^{2 r+1} Y$ be tangent vectors to $J^{2 r+1} Y$ at a point $x$. Expressing the value of the forms on both sides of (32) at $x$ and $\xi_{1}, \ldots, \xi_{n}$, we obtain

$$
\begin{aligned}
& \left(I_{k+1}\left(p_{k+1} \rho\right)-p_{k+1} \rho\right)\left(J^{2 r+1} \Xi_{k+1}(x), \ldots, J^{2 r+1} \Xi_{1}(x), \xi_{1}, \ldots, \xi_{n}\right) \\
& \quad=\frac{1}{k+1}\left(\mu_{\Xi_{1}}\left(J^{2 r+1} \Xi_{k+1}, J^{2 r+1} \Xi_{k}, \ldots, J^{2 r+1} \Xi_{3}, J^{2 r+1} \Xi_{2}, \xi_{1}, \ldots, \xi_{n}\right)\right. \\
& \quad-\mu_{\Xi_{2}}\left(J^{2 r+1} \Xi_{k+1}, J^{2 r+1} \Xi_{k}, \ldots, J^{2 r+1} \Xi_{3}, J^{2 r+1} \Xi_{1}, \xi_{1}, \ldots, \xi_{n}\right) \\
& \quad-\ldots \\
& \left.\quad-\mu_{\Xi_{k+1}}\left(J^{2 r+1} \Xi_{1}, J^{2 r+1} \Xi_{k}, \ldots, J^{2 r+1} \Xi_{3}, J^{2 r+1} \Xi_{2}, \xi_{1}, \ldots, \xi_{n}\right)\right) .
\end{aligned}
$$

It now follows that the $(k+1)$-contact part of the left-hand side of (33) vanishes, thus the $(n+$ $k+1$ )-form $I_{k+1}\left(p_{k+1} \rho\right)-p_{k+1} \rho$ belongs to $\Theta_{n+k+1}^{2 r+1} W$ (up to the canonical jet projection). In addition to this, it is easy to see that $p_{k+1} \rho-\rho \in \Theta_{n+k+1}^{2 r+1} V$ hence we conclude with $\mathscr{I} \rho-\rho \in$ $\Theta_{n+k+1}^{2 r+1} W$.

(b) Let $\rho \in \Omega_{n+k}^{r} W$ be an arbitrary $(n+k)$-form. From assertion (a) of this theorem we have $\mathscr{I} \rho-\rho \in \Theta_{n+k}^{2 r+1} V$. Suppose first that $\mathscr{I} \rho=0$. Then it follows that $-\rho \in \Theta_{n+k}^{2 r+1} W$. However, $\rho$ is defined on $W^{r} \subset J^{r} Y$ hence by definition (2) of contact forms we get $\rho \in \Theta_{n+k}^{r} W$.

Conversely, let $\rho \in \Theta_{n+k}^{r} W$. Then $\rho$ is expressible as a sum of the following terms

$$
\begin{aligned}
& A_{\sigma_{1} \ldots \sigma_{k+s}}^{I_{1} \ldots I_{k+s} i_{1} \ldots i_{s}} \omega_{I_{1}}^{\sigma_{1}} \wedge \cdots \wedge \omega_{I_{k+s}}^{\sigma_{k+s}} \wedge \omega_{i_{1} \ldots i_{s}} \\
& d\left(A_{\sigma_{1} \ldots \sigma_{k+s-1}}^{I_{1} \ldots I_{k+s-1} i_{1} \ldots i_{s}} \omega_{I_{1}}^{\sigma_{1}} \wedge \cdots \wedge \omega_{I_{k+s-1}}^{\sigma_{k+s-1}} \wedge \omega_{i_{1} \ldots i_{s}}\right)
\end{aligned}
$$

where $s=1,2, \ldots, n$. The summands $\mu$ of (34) for which $p_{k} \mu=0$, obey $\mathscr{I} \mu=0$ directly from the definition of $\mathscr{I}$. It remains to show that the condition $\mathscr{I} \mu=0$ is satisfied also for terms of the form

$$
\mu=d\left(A_{\sigma_{1} \ldots \sigma_{k}}^{I_{1} \ldots I_{I} i} \omega_{I_{1}}^{\sigma_{1}} \wedge \cdots \wedge \omega_{I_{k}}^{\sigma_{k}} \wedge \omega_{i}\right) .
$$

This was, however, already proved in Lemma 3 . Thus, the kernel of $\mathscr{I}$ coincides with the space of contact forms $\Theta_{n+k}^{r} W$.

(c) Finally, we show that $\mathscr{I}$ has the property of a projector, up to a lift of the canonical jet projection. From (a) we have $\mathscr{I} \rho-\rho \in \Theta_{n+k}^{2 r+1} W$, and property (b) of this theorem implies $\mathscr{I}(\mathscr{I} \rho-\rho)=0$. From linearity of $\mathscr{I}$ we conclude that $\mathscr{I}(\mathscr{I} \rho)-\mathscr{I} \rho=0$ for every $\rho \in$ $\Omega_{n+k}^{r} W$. This means that $\mathscr{I} \circ \mathscr{I}=\mathscr{I}$.

Remark 1 Theorem 1, (b), implies that the image of $\Omega_{n+k}^{r} W$ by $\mathscr{I}: \Omega_{n+k}^{r} W \rightarrow \Omega_{n+k}^{2 r+1} W$ is canonically isomorphic with the quotient group $\Omega_{n+k}^{2 r+1} W / \Theta_{n+k}^{2 r+1} W$. If $\rho \in \Omega_{n+k}^{r} W$, then the class $[\rho] \in \Omega_{n+k}^{2 r+1} W / \Theta_{n+k}^{2 r+1} W$ in the variational sequence can be identified with the globally defined differential form $\mathscr{I} \rho$. However, it may happen that a class of $\rho \in \Omega_{n+k}^{r} W$, an element of $\Omega_{n+k}^{s} W / \Theta_{n+k}^{s} W$ in the variational sequence of order $s, s<2 r+1$, is not a globally defined 
differential form (for an example, see [7]).

Remark 2 Instead of inductive definition (24) of operator $I_{k}$, it is also possible to use an equivalent definition by means of the operator $I_{1}$.

Let $k \geq 1$. Let $\rho$ be a $k$-contact $(n+k)$-form on $J^{r+1} Y$, and $\Xi_{1}, \Xi_{2}, \ldots, \Xi_{k}$ be arbitrary $\pi$ vertical vector fields on $Y$. We define a $k$-contact $(n+k)$-form $I_{k} \rho$ on $J^{2 r+1} Y$ by

$$
\begin{aligned}
& i_{J^{2 r+1} \Xi_{k}} \ldots i_{J^{2 r+1} \Xi_{2}} i_{J^{2 r+1} \Xi_{1}} I_{k} \rho=\frac{1}{k}\left(i_{J^{2 r+1} \Xi_{k}} I_{1}\left(i_{J^{r+1} \Xi_{k-1}} i_{J^{r+1} \Xi_{k-2}} \ldots i_{J^{r+1} \Xi_{3}} i_{J^{r+1} \Xi_{2}} i_{J^{r+1} \Xi_{1}} \rho\right)\right. \\
& \quad-i_{J^{2 r+1} \Xi_{k-1}} I_{1}\left(i_{J^{r+1} \Xi_{k}} i_{J^{r+1} \Xi_{k-2}} \ldots i_{J^{r+1} \Xi_{3}} i_{J^{r+1} \Xi_{2}} i_{J^{r+1} \Xi_{1}} \rho\right) \\
& \quad-i_{J^{2 r+1} \Xi_{k-2}} I_{1}\left(i_{J^{r+1} \Xi_{k-1}} i_{J^{r+1} \Xi_{k}} \ldots i_{J^{r+1} \Xi_{3}} i_{J^{r+1} \Xi_{2}} i_{J^{r+1} \Xi_{1}} \rho\right) \\
& \quad-\ldots \\
& \quad-i_{J^{2 r+1} \Xi_{2}} I_{1}\left(i_{J^{r+1} \Xi_{k-1}} i_{J^{r+1} \Xi_{k-2}} \ldots i_{J^{r+1} \Xi_{3}} i_{J^{r+1} \Xi_{k}} i_{J^{r+1} \Xi_{1}} \rho\right) \\
& \left.\quad-i_{J^{2 r+1} \Xi_{1}} I_{1}\left(i_{J^{r+1} \Xi_{k-1}} i_{J^{r+1} \Xi_{k-2}} \ldots i_{J^{r+1} \Xi_{3}} i_{J^{r+1} \Xi_{2}} i_{J^{r+1} \Xi_{k}} \rho\right)\right) .
\end{aligned}
$$

\section{Examples}

We conclude this paper with two examples of canonical representatives of differential forms, canonically isomorphic with classes in the variational sequence, well-known in the calculus of variations. From the variational sequence theory we observe that classes of forms represent Lagrangians ( $n$-forms), Euler-Lagrange expressions $((n+1)$-forms $)$, Helmholtz variationality conditions $((n+2)$-forms $)$, etc.

At first, consider a Lagrangian $\lambda$ of order $r$ on $J^{r} Y$, given in a fibred chart by $\lambda=\mathscr{L} \omega_{0}$ on $J^{r} Y$. $\lambda$ represents the class of a 1 -form (the Lagrange class), defined by its horizontal component. We find the canonical representative of the $(n+1)$-form $d \lambda$. By our definition of $\mathscr{I}$, we compute

$$
p_{1}(d \lambda)=p d \mathscr{L} \wedge \omega_{0}=\left(\sum_{l=0}^{r} \frac{\partial \mathscr{L}}{\partial y_{j_{1} j_{2} \ldots j_{l}}^{\sigma}} \omega_{j_{1} j_{2} \ldots j_{l}}^{\sigma}\right) \wedge \omega_{0},
$$

and obtain by Lemma 1, (15), the Euler-Lagrange form of $d \lambda$,

$$
\mathscr{I}(d \lambda)=I_{1}\left(p_{1} d \lambda\right)=E_{\sigma}(\mathscr{L}) \omega^{\sigma} \wedge \omega_{0},
$$

where

$$
E_{\sigma}(\mathscr{L})=\sum_{l=0}^{r}(-1)^{l} d_{j_{1}} d_{j_{2}} \ldots d_{j_{l}} \frac{\partial \mathscr{L}}{\partial y_{j_{1} j_{2} \ldots j_{l}}^{\sigma}}
$$

The mapping assigning to the Lagrange class its Euler-Lagrange expressions (36) represents the well-known Euler-Lagrange mapping.

Let $\varepsilon$ be a 1-contact, $\pi^{r, 0}$-horizontal form on $J^{r} Y$ (called the source form), an element of image of the Euler-Lagrange mapping. In a fibred chart $(V, \psi), \psi=\left(x^{i}, y^{\sigma}\right), \varepsilon$ is of the form $\varepsilon=\varepsilon_{v} \omega^{v} \wedge \omega_{0}$. We have

$$
p_{2} d \varepsilon=\sum_{l=0}^{r} \frac{\partial \varepsilon_{v}}{\partial y_{j_{1} j_{2} \ldots j_{l}}^{\sigma}} \omega_{j_{1} j_{2} \ldots j_{l}}^{\sigma} \wedge \omega^{v} \wedge \omega_{0} .
$$

By the definition of the operator $I_{2} \rho(24)$, for arbitrary $\pi$-vertical vector fields $\Xi_{1}, \Xi_{2}$ on $Y$ we 
get

$$
i_{J 2 r+1 \Xi_{2}} i_{J 2 r+1} \Xi_{1} I_{2} \rho=\frac{1}{2}\left(i_{J 2 r+1 \Xi_{2}} I_{1}\left(i_{J r+1} \Xi_{1} \rho\right)-i_{J^{2 r+1} \Xi_{1}} I_{1}\left(i_{J^{r+1} \Xi_{2}} \rho\right)\right) .
$$

Hence, we obtain a chart expression of $I_{2}\left(p_{2} d \varepsilon\right)$ of the form

$$
\begin{aligned}
\mathscr{I}(d \varepsilon) & =I_{2}\left(p_{2} d \varepsilon\right) \\
& =\frac{1}{2} \sum_{k=0}^{r} H_{v \sigma}^{j_{1} j_{2} \ldots j_{k}}(\varepsilon) \omega_{j_{1} j_{2} \ldots j_{k}}^{\sigma} \wedge \omega^{v} \wedge \omega_{0},
\end{aligned}
$$

where

$$
H_{v \sigma}^{j_{1} j_{2} \ldots j_{k}}(\varepsilon)=\frac{\partial \varepsilon_{v}}{\partial y_{j_{1} j_{2} \ldots j_{k}}^{\sigma}}-(-1)^{k} \frac{\partial \varepsilon_{\sigma}}{\partial y_{j_{1} j_{2} \ldots j_{k}}^{V}}-\sum_{p=k+1}^{r}(-1)^{p}\left(\begin{array}{l}
p \\
k
\end{array}\right) d_{j_{k+1}} d_{j_{k+2}} \ldots d_{j_{p}} \frac{\partial \varepsilon_{\sigma}}{\partial y_{j_{1} j_{2} \ldots j_{p}}^{V}} .
$$

$\mathscr{I}(d \varepsilon)$ is a globally defined object in the variational sequence, called the Helmholtz form, with coefficients (37), the Helmholtz expressions (cf. Krupka [9], Krbek and Musilová [4], Šeděnková-Volná [12]).

\section{References}

[1] ANDERSON, I. M.: Introduction to the variational bicomplex, Contemporary Math. 132 (1992), $51-73$.

[2] BAUDERON, M.: Le probleme inverse du calcul des variations, Ann. Inst. H. Poincaré, A 36 (1982), 159-179.

[3] DEDECKER P.-TULCZYJEW, W. M.: Spectral sequences and the inverse problem of the calculus of variations. In: Diff. Geom. Methods in Math. Phys., Proc. Conf., Aix-en-Provence and Salamanca 1979, Lecture Notes in Math. 836 (1980), 498-503.

[4] KRBEK, M.-MUSILOVÁ, J.: Representation of the Variational Sequence by Differential Forms, Acta Appl. Math. 88 (2005), 177-199.

[5] KRUPKA, D.: Some Geometric Aspects of Variational Problems in Fibred Manifolds, Folia Fac. Sci. Nat. Univ. Purk. Brunensis, Physica, XIV, Brno, Czechoslovakia, 1973, pp. 65., arXiv:math$\mathrm{ph} / 0110005$.

[6] KRUPKA, D.: Variational sequences on finite order jet spaces. In: Diff. Geom. Appl., Proc. Conf., Brno, Czechoslovakia, August 1989 (J. Janyška and D. Krupka, eds.), World Scientific, Singapore, 1990, pp. 236-254.

[7] KRUPKA, D.: Variational sequences in mechanics, Calc. Var. 5 (1997), 557-583.

[8] KRUPKA, D.-ŠEDĚNKOVÁ, J.: Variational sequences and Lepage forms. In: Diff. Geom. Appl., Proc. Conf., Prague, August 2004 (J. Bureš, O. Kowalski and D. Krupka, eds.), Charles University, Prague, Czech Republic, 2005, pp. 617-627.

[9] KRUPKA, D.: Global variational theory in fibred spaces. In: Handbook of Global Analysis (D. Krupka and D. Saunders, eds.) Elsevier, Amsterdam, 2007, pp 773-836.

[10] MIKULSKI, W. M.: Uniqueness results for operators in the variational sequence, Ann. Pol. Math. 95, No. 2 (2009) 125-133.

[11] ŠEDĚNKOVÁ, J.: On the invariant variational sequences in mechanics, Rend. Circ. Mat. Palermo, Proc. of the 22nd Winter School Geom. and Phys., Srni, January 2002; Ser. II, 71 (2003) 185-190.

[12] ŠEDĚNKOVÁ, J.: Representations of variational sequences and Lepage forms, Ph.D. Thesis, Palacky University, Olomouc, 2004.

[13] VOLNÁ, J.: Interior Euler-Lagrange operator, Preprint Series in Global Analysis and Applications, Palacky University, Olomouc, 6 (2005) 1-9.

[14] VITOLO, R.: Variational sequences. In: Handbook of Global Analysis (D. Krupka and D. Saunders, eds.) Elsevier, Amsterdam, 2007, pp. 1115-1163. 


\section{Jana Volná}

Department of Mathematics, Faculty of Applied Informatics, Tomas Bata University in Zlin

Nad Stranemi 4511, 76005 Zlin, Czech Republic

e-mail:volna@fai.utb.cz

\section{Zbyněk Urban}

Lepage Research Institute, 78342 Slatinice, Czech Republic

e-mail: zbynek.urban@lepageri.eu 